\title{
Article
}

\section{Independent advocacy for children and young people: developing an outcomes framework}

Thomas, Nigel, Crowley, Anne, Moxon, Daniel, Ridley, Julie, Street, Cathy and Joshi, Puja

Available at http://clok.uclan.ac.uk/17042/

Thomas, Nigel ORCID: 0000-0002-5310-9144, Crowley, Anne, Moxon, Daniel, Ridley, Julie ORCID: 0000-0002-0879-308X, Street, Cathy and Joshi, Puja (2017) Independent advocacy for children and young people: developing an outcomes framework. Children \& Society. ISSN 0951-0605

It is advisable to refer to the publisher's version if you intend to cite from the work. http://dx.doi.org/10.1111/chso.12207

For more information about UCLan's research in this area go to http://www.uclan.ac.uk/researchgroups/ and search for <name of research Group>.

For information about Research generally at UCLan please go to http://www.uclan.ac.uk/research/

All outputs in CLoK are protected by Intellectual Property Rights law, including Copyright law. Copyright, IPR and Moral Rights for the works on this site are retained by the individual authors and/or other copyright owners. Terms and conditions for use of this material are defined in the policies page.

\section{CLoK}

Central Lancashire online Knowledge www.clok.uclan.ac.uk

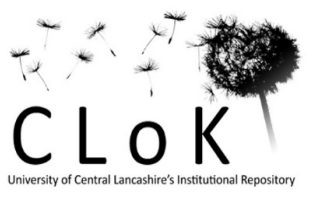




\title{
Independent advocacy for children and young people:
}

\author{
developing an outcomes framework
}

Nigel Thomas, Anne Crowley, Dan Moxon, Julie Ridley, Cathy Street and Puja Joshi

\section{Introduction}

Advocacy services for vulnerable children and young people began to be provided in the 1980s (Willow, 2013) and have grown as legislation and guidance expanded the range and remit of services (Wood and Selwyn, 2013). Research has followed the development of policy and services, but until recently has not examined the impact and outcomes of advocacy in any depth. In this article we draw on findings from a study of the outcomes and impact of independent advocacy for children and young people to explore how the value of advocacy is understood by them and by advocates, social workers and other professionals, and to consider what differences advocacy can make to the lives of children and young people (Thomas et al., 2016). Our findings indicate that the outcomes of advocacy in children and young people's lives can be significant and wide-ranging, including both direct effects on the child or young person and wider impact on services. This complex picture has implications for how best to capture and report the outcomes of advocacy, which we explore in the latter part of the article with the aid of a proposed new conceptual framework.

\section{Background}

The Department for Education and Skills described advocacy as follows:

Advocacy is about speaking up for children and young people and ensuring their views and wishes are heard and acted upon by decision makers. (DfES, 2004, p. 8)

Other definitions put more emphasis on enabling children and young people to speak up for themselves about matters that concern them (Dalrymple and Hough, 1995). However, it is generally accepted that advocacy involves listening and empowering a child or young person by helping them to represent their views, supporting them and protecting their rights through a child-led approach (Oliver et al., 2006; Thomas, 2008; Moss, 2011). Currently, children and young people are legally entitled to advocacy if they are looked after or in need, receiving mental health services (in particular in-patient services) or detained in the youth justice system (Brady, 2011). ${ }^{1}$ Despite

\footnotetext{
${ }^{1}$ The Children Act 1989 introduced the right of children in care or in need to participate in decisions that affect them and to make complaints. These rights were extended to care leavers by the Children (Leaving Care) Act 2000, and the Adoption and Children Act 2002 placed a statutory duty on local authorities to provide looked after children, care leavers and children in need with assistance when making, or intending to make, a complaint, constituting a legal entitlement to advocacy. The Secure Centre Training Rules 1998 give young people the right to access advocacy support and representation from an independent advocate while they are in custody. The Health and Social Care Act 2001 places a duty on the Secretary of State for Health to make
} 
these statutory provisions, children and young people's access to advocacy is uneven, often being subject to local spending decisions rather than being needs-driven (Oliver, 2008a).

Advocacy services for children and young people have developed in a largely ad hoc manner with some parts of the country being particularly well-resourced and others having very limited provision (Pona and Hounsell, 2012). A number of studies have shown that the current state of outcomes monitoring in the advocacy sector is inconsistent at best, with commissioners making individual decisions about what information they require from advocacy providers. This often tends to focus on measurable outputs (e.g. the number of people accessing advocacy) rather than outcomes (including the effects on children and young people's lives). Output data is easier to capture, and until recently this has been enough for many funders, but it tells us nothing about the impact of advocacy on service users (Newbigging et al., 2015). Without robust evaluation, the effectiveness of advocacy is unclear. Until now, more attention has been paid to researching the principles of advocacy and establishing definitions than to seeking evidence for the impact of advocacy (Oliver, 2008b).

Over the last decade there has been an emphasis on collecting outcomes data across health and social care services for children and young people, with an accompanying literature exploring the use of outcomes tools that aim to capture changes in children and young people's health and wellbeing. Government Departments and public bodies have produced a variety of reports highlighting the importance of outcomes data, while a range of independent bodies have produced guides on this topic (see New Economics Foundation, 2009; Nevill and Lumley, 2011; Gutman and Vorhaus, 2012; Law and Wolpert, 2014; Department of Health and NHS England, 2015; Children and Young People's Mental Health and Wellbeing Taskforce, 2015; Children's Services Development Group and Local Government Information Unit, 2015; Anna Freud Research Centre and Public Health England, 2016).

Knowing about outcomes is important in creating 'feedback loops' for practitioners to help them improve children and young people's lives. Two key planks of the Children and Young People's IAPT (Improving Access to Psychological Therapies) programme are the use of outcomes tools and children and young people's participation, and the programme strongly advocates the use of outcomes tools as a way of promoting dialogue with young people and involving them in their care. In the words of a CAMHS psychologist quoted by the Children and Young People's Mental Health and Wellbeing Taskforce:

"If data collects meaningful information that can be useful for clinicians and patients alike to monitor their progress, data collection becomes part of the therapy."

The absence of good outcomes data makes it difficult to make judgements about the value of advocacy or to make the case for more comprehensive services. This is especially significant during

advocacy available to anyone (including children and young people) who wishes to make a complaint about their NHS care; the Mental Health Capacity Act 2005 places a duty on local authorities to provide an Independent Mental Capacity Advocate (IMCA) for people aged 16 years or older who have nobody else to represent them and who lack the capacity to make decisions about certain issues; and the Mental Health Act 2007 provides for all people who are 'sectioned' under the Act to access the support of an Independent Mental Health Advocate (IMHA), regardless of age. 
the current period of 'austerity' in the UK where demands for public expenditure, including statutory requirements, have to compete for scarce resources (Centre for Local Economic Strategies, 2014). As independent advocacy services are also subject to competitive tendering, providers have found that evaluating the impact of their work is becoming more important to securing contracts with local authorities (Rapaport et al., 2006). However, Brady (2011) observed that, in children and young people's advocacy, monitoring and evaluation was still carried out on a piecemeal basis, often not taking all stakeholders' views into account; local authorities do not currently collect systematic data from their providers, nor is there much guidance regarding what or how information should be collected and monitored. Unless advocacy providers can effectively monitor their own work, their services may be especially vulnerable in the current economic climate, which may be a time when they are needed more than ever. For example, our research suggests that failure of hard-pressed social workers to discharge their duties increases the importance of independent advocacy in helping to ensure that decision-making actively considers the wishes and feelings of the child.

It is therefore doubly important that advocacy providers, commissioners and researchers find ways to overcome the challenges that currently face the sector in relation to outcomes monitoring. To facilitate progress, it is necessary to explore current understandings of the outcomes and impact of advocacy and review the methods currently used for capturing and monitoring data, and this is what our study aimed to do. This paper presents findings that show clearly the value that is placed on advocacy and what it achieves from the perspective of the different stakeholders, with further analysis to produce a framework for categorising the various outcomes and impacts. There is impressive evidence in personal stories of the positive impact of independent advocacy, and we hope that our research will help the sector to consider afresh how these positive impacts can be represented in effective outcomes monitoring and reporting.

We should be clear that this is not an outcomes study in itself, rather an examination of what advocacy services currently do in terms of outcomes measurement, and what young people and professionals consider to be important outcomes. We hope it can be the basis of a collaborative endeavour to create consensus around what would be meaningful outcomes to measure in advocacy for children and young people, creating the shared knowledge and tools that are needed for the measurement of outcomes to be possible, and relevant.

\section{Methodology}

This paper is based on research conducted by The Centre for Children and Young People's Participation at the University of Central Lancashire with the National Children's Bureau (NCB) Research Centre in 2015, for the Children's Commissioner for England (Thomas et al., 2016).

An online survey was distributed to a range of children's advocacy providers across England and was completed by 38 organisations. It asked about what information on outcomes was recorded, how the information was used, and how children and young people were involved in the evaluation of services. ${ }^{2}$ From this six advocacy services were chosen for closer study, reflecting a range of

\footnotetext{
${ }^{2}$ Details of the survey results are given in Thomas et al. (2016).
} 
practices and settings. Four were local authority services (one provided 'in-house' and three contracted to independent providers, while the other two were in a young offender institution and a mental health service. The sample included several of the main national advocacy providers.

Within each site we examined recording and monitoring systems, and interviewed advocates and advocacy team managers $(n=19)$ in addition to other professional stakeholders such as commissioners of services, social workers, independent reviewing officers, prison governors and clinical staff $(n=19)$. Children and young people were invited to take part in either an individual interview or a focus group with others from the same site; 17 young people took part in interviews, 16 in focus groups ${ }^{3}$. Initial analysis produced a field report for each site, and was then combined into a thematic analysis across all six sites.

The following working definitions of output, outcomes and impact were adopted:

Outputs are measures of activity such as cases taken, time spent, types of issue presented, demographic information - information that we expected to be a key part of recording systems, but not directly relevant to our research questions.

Outcomes are the consequences of advocacy for children and young people, such as issue resolution, feeling heard, and user satisfaction, which can in principle be identified during or at the conclusion of an episode of advocacy however defined. Outcomes of this kind were the principal focus of our research.

Impact we understood to mean the more general effects of advocacy provision on services as a whole. This was also a focus of our research.

Ethical approval for the project was given by the University of Central Lancashire, following approval by the Health Research Authority and the National Offender Management Service; additional permissions were obtained from the Youth Justice Board, the Association of Directors of Children's Services (ADCS), individual local authorities and an NHS Trust.

\section{The value of advocacy to young people}

As with Oliver et al. (2006), this study suggested generally high levels of satisfaction amongst young people who use advocacy services; all the young people who participated in the study placed considerable value upon advocacy support. Some primarily valued advocates when they helped them to achieve their goals and resolve specific issues. However, the majority valued the relationship irrespective of whether or not the advocate helped bring about a change the young person wanted. Some understood that an advocate could not always achieve resolution of an issue to a young person's satisfaction, and were appreciative of advocates' open and frank approach to what was possible and realistic, especially as they felt let down by other professionals who failed to offer any explanation when they did not deliver on promises made. This speaks about the relational quality of the advocacy partnership, and the importance of open and honest communication with young people (Boylan and Dalrymple, 2009). In line with previous research,

\footnotetext{
${ }^{3}$ Participants were aged 11-21. The gender balance varied between sites, with a slight female predominance overall. Five of the sample were Black or mixed-heritage, and two were disabled.
} 
young people from all six sites recognised 'being listened to' as a key outcome of advocacy, and for some this was regardless of whether the issue they wanted help with was resolved as they wished. Given the numbers of young people who participated in the research, it is not possible to identify significant differences between children and young people from different backgrounds.

\section{Defining the outcomes of independent advocacy}

Although the general value of advocacy was clear to all participants, defining the changes and outcomes that occurred as a result of it was considerably more complex. Different framings of advocacy outcomes were to some extent associated with the category of research participant (young people, advocates, professionals). However, there was variation within these groups and also between sites, which rules out making simple generalisations. Accordingly, whilst there was a broad consensus about the importance of advocacy achieving the practical change the young person wanted, the relative importance of this varied considerably.

Although young people emphasised issue resolution and practical changes as important, they also spoke of achieving process outcomes such as 'getting their voices heard' and having a greater involvement in decision-making, as well as personal development outcomes such as improved selfconfidence and increased ability to self-advocate. Young people in secure mental health and youth offender settings mainly talked of advocacy in terms of 'getting their voices heard' or helping them communicate their wishes and feelings, and of improving their relationship with services, whilst those in the care and protection settings tended to have higher expectations of 'getting a result'. This may be seen to reflect the greater relative powerlessness of young people and their advocates in secure settings.

Like Oliver et al (2006), we found that in general adults were more likely than young people to highlight individual empowerment and 'young people having the opportunity to express their views' as the most important and relevant outcomes to consider; some advocates and external stakeholders also framed outcomes in terms of young people's personal development and enhancing their capacity for self-advocacy. Three distinct outcome themes were identified, whose relative importance varied across sites and types of participant:

- Improving participation and 'giving young people a voice';

- Achieving change and resolving issues identified by the young person;

- Personal growth and development for young people.

\section{Improving participation and 'giving young people a voice'}

'Giving children and young people a voice', in other words framing advocacy as supporting young people to articulate their wishes and feelings and ensuring that they were heard, was the most commonly identified outcome of advocacy. Young people reported that having an advocate had primarily helped to improve their involvement and participation in various official processes.

“...somebody there that could help interpret what I'm trying to say because sometimes I put things forward in a very kind of complicated way that may not necessarily be what I 
want to put forward. I don't like meetings because I might say one thing but mean another....the advocate makes sure it is what I do want to say at the meetings..." (Young person)

'Giving children and young people a voice' appeared to include several distinct elements:

- Supporting children and young people to articulate their wishes and feelings;

- Helping them to 'put it across in the right way';

- Making sure that adults listened and took young people's views seriously;

- Enabling children and young people to feel listened to;

- Enabling them to be part of the decision-making process.

A critical outcome identified by all types of participant was that young people were listened to and were more involved in the decisions affecting their lives. In other words, advocacy increased young people's sense of agency.

"She has helped me a lot, and I felt like I was an outcast in meetings and like, 'cause I didn't know how to say my words, everything like that. I felt like a little person.... so she showed me how to stand out and really they listened to me more." (Young person)

\section{Achieving practical change and resolving issues}

For many research participants, the most important outcome was to achieve the practical change the young person wanted from advocacy, to find a resolution to their concerns. As one professional put it, "The resolution is the important thing because if we have resolved their issues then by de facto [sic] they have been heard."

Practical change was an outcome welcomed by young people, even when the change was not what they had originally asked for. Practical outcomes achieved by young people with advocacy included changes in contact or living arrangements, education, pocket money, curfew or travel restrictions, and more generally changes in how they were treated by service providers and in their relationships with social workers and carers.

Advocates and stakeholders acknowledged that defining outcomes solely in terms of issue resolution was too limited. First, in practice it was not always possible to achieve the change sought, for reasons outside the control of the advocate. Second, some young people did not achieve their desired outcomes but still reported positive experiences of advocacy. Understanding why the desired result was not achieved, and receiving a full explanation, was seen by many young people as a positive outcome.

Despite advocates' best efforts, in some situations (particularly in secure settings) issue resolution was not possible. Instead, ensuring that a young person understood what choices were possible, and what kinds of changes were achievable and why, was seen as key to effective advocacy. A 'good enough' outcome might be that advocacy ensures that young people's voices are heard, even if the issue is not resolved to their satisfaction. For some advocates, the primary question was whether the young person was happy with the actual resolution, regardless of whether it was what they had originally asked for: 
"I think my main question would be was the young person happy with [the way the issue was resolved], because the outcome might not necessarily be what they wanted it to be the first place." (Advocate)

\section{Personal growth and development}

The third way in which outcomes were framed was as personal change and development for the young person: for example, increasing self-confidence and self-esteem. Ultimately this could mean the confidence to articulate one's own needs and to self-advocate - to 'fight my own battles' and 'talk for myself:

"Our aim as advocates is to ensure that young people can get as far along the road to self-advocacy as possible. Some of them will never be able to achieve that. But to me the greatest outcome for my work is having a young person say, 'Do you know what, actually I think I can do this on my own. I don't need you any more'. And that feels brilliant.” (Advocate)

This might mean young people learning about how the 'system' works, or being informed about their rights and entitlements; or it might mean that participation empowered young people so that they were better able to manage similar challenges in the future. Personal growth could include self-confidence, self-efficacy, self-esteem, or communication and interpersonal skills. In youth justice settings it also included developing emotional control to resolve issues without use of violence or aggression. For some young people, especially in mental health settings, advocacy could provide validation through feeling that they were worth something, their voice was worth hearing, and that professionals listened and took them more seriously. To one young person in local authority care the advocate "made me feel like I was worth something, not just dashed around like some paperwork filled in and nothing else; [they] cared about how I felt and what my opinions were and made me feel...calm." Adult participants also spoke of longer-term outcomes that included life skills, self-esteem, independence, and empowerment.

Some advocates suggested that young people were only aware of the tangible outcomes:

"I might say that for me the outcome is that they've learnt to voice their wishes more, they're more confident in meetings, in challenging decisions. They won't think of that. It's 'Oh I got a new social worker' or 'I got a phone'. They won't be thinking 'well I stuck to my guns and I got what I asked for and eventually made a good case for it', which is all useful life skills isn't it?" (Advocate)

However, the young people who spoke to us were often highly aware of these kind of benefits:

"The main thing is you go to an advocate for a reason and want a good outcome, but they can also teach you ways to deal with your problems. So you feel more confident not just in care but as you grow up in life." (Young person) 


\section{Wider impact of independent advocacy}

The research also provided many examples of the wider impact of advocacy support on the ways in which services operate. We may categorise these impacts as:

- changes to decision-making culture;

- changes to policies, practices and services more broadly.

Notwithstanding the practical difficulties of capturing or studying these wider impacts, our research suggests that they can be significant and should be considered a key indicator of the value of advocacy.

\section{Impact on decision-making culture}

For both advocates and stakeholders, a key impact was on decision-making culture, in putting children and young people at the centre of decision-making processes so that their views and wishes are taken into account.

Stakeholders reported that advocacy had enabled young people's direct involvement to shape discussions with professionals. Young people were no longer talked about and decisions made for them, but took a more active role in decision-making. This particularly applied to child protection conferences and looked after children (LAC) reviews, where advocates, independent reviewing officers (IROs) and conference chairs reported that young people had become more central. In one site a child protection chair explained how an advocate's presence can help shape the focus of a conference, the nature of the discussions, the way decisions are made and the impact on the child or young person's life:

"Having an advocate in conference - it changes the focus of the meeting... One of the challenges as a conference chair is to keep everybody focused on the child that you're there to discuss. Having the child in the room is by far the best way to do that. Having an advocate there on behalf of the child is like the second best to that... I have had some really powerful moments in conference where children and young people, supported by advocates, have been able to say how things are from their perspective. I have also had a number of experiences where advocates have spoken the child's words... when the child's own words are used and not interpreted by someone..., it has a real impact in conference and has really shifted a number of cases." (Stakeholder)

\section{Impact on policies, practices and wider service operations}

Feedback from advocates working with children and young people suggests that independent advocacy can draw attention to gaps in provision or to poor practice. Stakeholders and advocates provided many examples of how issues raised by individual young people through advocacy had led to long-term changes in services, by prompting providers to review how services are delivered and to embed opportunities for children and young people to share their views and experiences. 
A stakeholder in one site told how young people, with the support of advocates, complained that taxis organised by the local authority (to pick them up for school or family contact) were frequently late or did not show up. As a result the local authority reviewed the contract and established a mechanism for young people to report future problems.

Problems were often identified through an individual case and then taken up as it became clearer that the issue affected other young people. Advocates were well placed to notice patterns and discuss them with service managers. In some sites advocates linked directly with accountability mechanisms such as the children in care council.

Other examples of changes included the very practical (such as access to satellite television in a care home) and the systemic (improvements in care planning and placement arrangements). There were also instances of advocacy impacting on the process of policy making: stakeholders and advocates reported how involvement of children and young people had become standard practice and gave examples of staff being more aware of ensuring that service user voices are heard:

"What advocates do really well is make the voice of the young people absolutely central and bring everyone back to that... I've seen that have such an impact, so many times. It helps the adults be more child-centred. They all have their agenda - the social worker, the teacher, the parent. Advocacy brings it back to the child." (Advocate)

In such cases, advocacy appeared to have shifted providers' perceptions of children and young people from passive recipients of services to users with entitlements, with recognition that if entitlements were denied services would be accountable. Young people reported that advocacy had helped them to use complaints procedures to hold service providers to account; an impact they described as 'ensuring things are done properly' or helping you 'get what you are entitled to' (see Pithouse and Crowley, 2007).

Advocacy services were also seen by professionals as adding value to services by supporting children and young people to understand what to expect, their entitlements, the processes involved and how to navigate them. Information collected by advocates was considered valuable 'intelligence' that could help to improve policy and practice. For example, in youth justice advocates felt that their work had helped to reduce reoffending and improve successful resettlement rates, for example by assisting young people to access housing support services.

However, whilst the research identified many such examples of the wider impact of advocacy, these were not systematically recorded in any of the six sites.

\section{Developing an outcomes framework}

This research confirms the value of independent advocacy in supporting children and young people's entitlement to quality services, their rights to protection from harm and abuse and to participate in decisions about their lives. It illustrates the breadth of individual outcomes, and substantial wider impacts, that advocacy can produce. As we have noted, this complex picture creates challenges for advocacy providers and commissioners in deciding what outcomes of independent advocacy should be captured, recorded and analysed. 
The value of independent advocacy demonstrated, particularly by the testimonies of young people who participated in this study, suggests that, far from being an 'added extra', advocacy is now playing an indispensable role in delivering positive outcomes for children and young people. This is well received by service users, especially in circumstances where they have fractured or nonexistent relationship with their case- or key-worker. In some of the sites, the relationship between advocates, social workers and other professionals suggested a respect for the role of the advocate as offering much more than just support for the child or young person to speak out.

There is a need for advocacy services, commissioners and researchers to collaborate to overcome the challenges that face the sector in capturing evidence of outcomes and wider impact and demonstrating the value of advocacy. Advocacy providers should together develop a consistent approach to monitoring outcomes that will not over-burden front-line workers or conflict with underlying values. This is a challenge, as this research confirmed; the competitive market in advocacy provision means that agencies are often reluctant to share practices (see also Wood and Selwyn, 2013).

The starting point must be a common framework for understanding outcomes. As noted earlier, we began with a rough working conceptualisation of outputs, outcomes and impact. From the research we can conclude that the definitions of outcomes and impact need to be broken down, in order to accommodate and reflect the longer-term effects on the individual child or young person of the advocacy experience, which may be regarded as outcomes (but a different kind of outcome, challenging to capture and record) or as impact (but an individual rather than a general impact, still challenging to capture and record). We therefore distinguish between:

(i) outcomes for individuals linked to the specific aims of advocacy - which include items related to issue resolution as well as enabling children and young people to have a voice;

(ii) impact on individuals such as growth in confidence and skills as a result of the experience of advocacy; and

(iii) wider impact on other children, services, policies and professional cultures.

Young people consulted by the Office of the Children's Commissioner on the findings of this study engaged readily with this threefold typology and were able to allocate specific outcomes to these categories, although they also found some overlaps (Thomas et al., 2016).

Once this typology is established, different types of outcomes and impact can be identified within the categories, as set out in Figure 1. This could, we suggest, form the basis for a national system to understand, record and analyse the outcomes and impact of independent advocacy for children and young people. 
Figure 1: Outcomes and Impact of Independent Advocacy: A Typology

\section{Outcomes for individuals}

\section{Issue resolution outcomes:}

Issue resolved as initially identified by the young person

$>$ Issue resolved in a different way, but to the young person's satisfaction

$>$ Issue not resolved to the young person's satisfaction (if negative outcomes are included)

Process outcomes:

Young person felt listened to

$>$ Young person felt they contributed to decision-making

$>$ Young person had a better understanding of processes

$>$ Young person had a better understanding of their rights

\section{Satisfaction outcomes:}

$>$ Young person would use advocacy service again

$>$ Young person would be confident to self-advocate in similar situation in future, knowing that the advocacy service was there to fall back on

Young person would recommend service to others

Personal outcomes:

Young person feels better about themselves as a result of the advocacy process

$>$ Young person feels more confident in speaking up as a result of the advocacy process

$>$ Young person has developed new skills as a result of the advocacy process

Relationship outcomes:

Young person had better relationship with service providers following the advocacy process

\section{Impact on individuals}

Young person has grown in confidence, at least in part as a result of experience of advocacy

$>$ Young person has grown in skills, at least in part as a result of experience of advocacy

$>$ Young person has grown in self-esteem, at least in part as a result of experience of advocacy

\section{Wider impacts}

Identifiable changes in service provision attributable to work of advocacy service

$>$ Identifiable growth in children and young people's participation attributable to work of advocacy service

$>$ Identifiable shift in professional culture attributable to work of advocacy service

$>$ Identifiable changes in patterns of complaints attributable to work of advocacy service

$>$ Identifiable changes in local policy attributable to work of advocacy service

$>$ Identifiable changes in national policy attributable to work of advocacy service 
Adopting a common framework or common language for understanding and categorising the outcomes of independent advocacy is an important step towards instituting mechanisms for capturing evidence of those outcomes, which could underpin a range of different tools and monitoring systems. Some of the outcomes and impacts in our typology are easier to capture than others, as we have learned from this research. We are unsure whether longer-term impacts for individual children or young people can be captured in a comprehensive way, and we found no example of this being successfully done. However, there are sampling approaches that may enable some of these impacts to be detected, if resources can be made available. Other individual outcomes are all in principle capturable during or at the conclusion of an episode of advocacy, subject to all the difficulties and challenges identified in this research. It is clear, from what advocates, professionals and especially young people told us, that identification of these outcomes has to be done in collaboration with children and young people, and therefore that whatever methods are used must be 'user-friendly'.

Identifying the wider impacts of advocacy presents different challenges, since they are not 'casebased' or dependent on individual evaluations, and will require close collaboration between providers and commissioners, possibly based around the processes of quarterly and annual reporting which we found to be common. If providers and commissioners are able to work together to devise efficient and user-friendly approaches to these tasks, there is a good chance of overcoming some of these challenges. Researchers also have a continuing contribution to make: a robust approach to monitoring and reporting the outcomes of independent advocacy has great potential benefits for those providing the service, those commissioning it, those receiving it and also for those who study it.

\section{Conclusion}

This study has provided insights into the value of advocacy and the difference it can make to the lives of children and young people. Whilst challenges remain, the case for routinely capturing and reporting on the outcomes and wider impact of advocacy services in a coherent and comparable way is compelling. The assumption that advocacy is a self-evidently worthwhile is no longer sufficient to convince funders; it must be seen to make a difference to the lives of children and young people.

A common framework of outcomes and impact, and a consistent national system for recording and measuring these, would enable greater consistency of service provision for children and young people, especially those who move between services. It would also make it easier for all involved - commissioners, providers and policy-makers - to compare the effectiveness of different services. However, it is important to retain a space in which advocacy providers can offer something distinctive and innovative, and it is clear that progress towards any kind of standard framework can only be made if providers and commissioners work together, and do this in co-production with young people. 


\section{References}

Anna Freud Research Centre and Public Health England (2016) Measuring and monitoring children and young people's mental wellbeing: a toolkit for schools and colleges. London: Public Health England

Boylan, J. and Dalrymple, J.(2009) Understanding Advocacy for Children and Young People.

Maidenhead: Open University Press

Brady, L. (2011) Where is my advocate? A scoping report on advocacy services for children and young people in England. London: Office of the Children's Commissioner.

Centre for Local Economic Strategies (2014) Austerity Uncovered: Final report presented to the TUC. London: TUC.

Children and Young People's Mental Health and Wellbeing Taskforce (2015) Data and Standards Task and Finish Group Report: Children and Young People's Mental Health and Wellbeing Taskforce. London: Department of Health.

Children's Services Development Group And Local Government Information Unit (2014) Collaborating for Better Outcomes: Final Report from the Children's Services Taskforce. London: Local Government Information Unit.

Crowley, A and Pithouse, A (2007) 'Adults Rule? Children, advocacy, and complaints to social services'. Children \& Society 21(3), 201-213.

Dalrymple, J. and Hough, J. (1995) Having a voice: An exploration of children's rights and advocacy. Birmingham: Venture Press/British Association of Social Workers.

Department of Health and NHS England (2015) Future in mind: promoting, protecting and improving our children and young people's mental health and wellbeing. London: Department of Health.

Gutman, L. and Vorhaus, J. (2012) The Impact of PupilBehaviour and Wellbeing on Educational Outcomes Research Report: DFE-RR253. London: Department for Education

Law, D. and Wolpert, M. (2014) Guide to Using Outcomes and Feedback Tools with Children, Young People and Families. London: Child Outcomes Research Consortium.

Moss, C. (2011) Safeguarding looked after children through advocacy: Looked after children's views on advocacy. London: NSPCC.

Nevill, C. and Lumley, T. (2011) Measuring together: Impact measurement in the youth justice sector. London: New Philanthropy Capital.

New Economics Foundation (2009) A Guide to Commissioning Children's Services for Better Outcomes. London: New Economics Foundation.

Newbigging, K., Ridley, J., McKeown, M., Sadd, J., Machin, K., Cruse, K., De La Haye, S., Able, L. and Poursanidou, K. (2015) Independent Mental Health Advocacy: The Right to be Heard. London: Jessica Kingsley Publishers. 
Oliver, C. (2008a). 'Setting the scene: Funding, patterns of advocacy provision and children's access to advocacy services' in Oliver, C. and Dalrymple, J. (eds) Developing advocacy for children and young people: Current issues in research, policy and practice. London: Jessica Kingsley. [26-42]

Oliver, C. (2008b). 'The Impact of Advocacy' in Oliver, C. and Dalrymple, J. (eds) Developing advocacy for children and young people: Current issues in research, policy and practice. London: Jessica Kingsley. [184-203]

Oliver, C., Knight, A. and Candappa, M. (2006) Advocacy for looked after children and children in need: Achievements and challenges. London: Thomas Coram Research Unit, Institute of Education, University of London.

Pithouse, A. and Crowley, A. (2007) Adults Rule? Children, Advocacy and Complaints to Social Services, Children \& Society 21(3), 201-213.

Pona, I. and Hounsell, D. (2012) The value of independent advocacy for looked after children and young people. London: The Children's Society.

Rapaport, J., Manthorpe, J., Hussein, S., Moriarty, J. and Collins, J. (2006) 'Old issues and new directions: Perceptions of advocacy, its extent and effectiveness from a qualitative study of stakeholder views'. Journal of Intellectual Disabilities 10(2), 191-210.

Thomas, N. (2008) 'Consultation and Advocacy' in Luckock, B. and Lefevre, M. (eds) Direct Work: Social work, with children and young people in care. London: British Association for Adoption and Fostering. [97-114]

Thomas, N., Street, C., Ridley, J., Crowley, A., Moxon, D., Joshi, P., Amalathas, E., Rix, K. and Edwards, A. (2016) Independent Advocacy: Impact and Outcomes for Children and Young People. London: Children's Commissioner.

Willow, C. (2013) Independent Advocacy in Child Protection: Guidance for Policy Makers. London: National Children's Bureau.

Wood, M. and Selwyn, J. (2013) The Characteristics of young people using independent advocacy services. Bristol: The Hadley Centre.

\section{Acknowledgements}

The research presented here was commissioned and funded by the Children's Commissioner for England, who retains intellectual property in the data. The authors would like to thank the staff of the Office of the Children's Commissioner, the members of the project advisory group, the agencies who responded to the survey, and especially the advocates, other professionals and young people in the six study sites who gave so generously of their time. 